\title{
Heterogeneous InP on SOI Integration for the Realization of All-Optical Logic Devices
}

\author{
G. Morthier ${ }^{1 *}$, L. Liu ${ }^{1 * *}$, R. Kumar ${ }^{1}$, P. Méchet ${ }^{1}$, K. Huybrechts ${ }^{1}$, G. Roelkens ${ }^{1,2}$, T. Spuesens ${ }^{1}$, T. De Vries ${ }^{2}$, E-J. \\ Geluk $^{2}$, P. Regreny ${ }^{3}$, R. Baets ${ }^{1}$, D. Van Thourhout ${ }^{1}$ \\ 1. Photonics Research Group, INTEC Department, Ghent University-IMEC, B-9000 Gent, Belgium \\ 2. COBRA Research Institute, Technische Universiteit Eindhoven, Postbus 513, $5600 \mathrm{MB}$ Eindhoven, The Netherlands \\ 3. Université de Lyon; Institut des Nanotechnologies de Lyon INL-UMR5270, CNRS, EcoleCentrale de Lyon, Ecully, F-69134, France \\ *E-mail: Geert.Morthier@intec.ugent.be, **Currently at DTU Fotonik, Denmark.
}

\begin{abstract}
InP-based microdisk lasers, heterogeneously integrated onto SOI waveguides, can be used as generic non-linear devices for realizing all-optical logic. We will discuss the performance of individual microdisk lasers, and the implementation of more complex circuits.

(C) 2009 Optical society of America

OCIS-codes: (060.6719) Packet switching; (250.3750) Optical logic devices; (250.4745) Optical processing devices
\end{abstract}

\section{Introduction}

With the ever increasing data rates in telecom and datacom networks, as well as in different short distance interconnections, there is an increasing need for all-optical packet switching implementations to overcome the bottlenecks imposed by the speed limitations of current microelectronic processors. While optical transport of data has a clearly proven advantage over electrical transport, the same cannot be said for the switching, processing or buffering of signals. Indeed, many previously proposed solutions for all-optical processing of digital signals have one or more significant drawbacks as compared to their electronic counterparts. A high power consumption is probably the main drawback of many all-optical signal processing techniques [1], but several proposed solutions are also complex and bulky set-ups, which could hardly be transformed into real products, let alone be fabricated as a small footprint photonic integrated circuit or PIC.

Using the silicon-on-insulator material platform with its high refractive index contrast, it is possible to make very compact passive optical waveguide circuits. In addition, by heterogeneously integrating Indium Phosphide membranes, it is possible to implement high speed, low-power and compact active devices such as microdisk laser diodes and resonators [2]. Recently, we have demonstrated compact, electrically pumped microdisk lasers with diameters as small as $5 \mu \mathrm{m}$ using the III-V/SOI heterogeneous integration [3]. Using optimized microdisk lasers, we also demonstrated the first electrically pumped all-optical flip-flops (AOFF) on the SOI platform [4]. Other functionalities such as gating and wavelength conversion have also been demonstrated [5-6]. The variety of functionalities that can be obtained using these microdisk lasers and the fact that they can easily be coupled to compact passive SOI optical circuits, makes them very suitable as basic building block to implement more elaborate photonic integrated circuits implementing more complex optical logic.

In this paper, we will give an overview on the use of these heterogeneously integrated microdisk lasers for alloptical signal processing. The implementation of new logic devices will be discussed and we will also report on the first photonic integrated circuits that comprise several heterogeneously integrated microdisks. These PICs are currently being fabricated.

\section{Fabrication and device concepts for AOFFs, gates and wavelength converters}

The InP microdisk lasers are heterogeneously integrated onto SOI using adhesive die-to-wafer bonding with the divinylsiloxane-benzocyclobutene (DVS-BCB) polymer [3]. The SOI circuit, with a 220nm top silicon layer and a 2 $\mu \mathrm{m}$ buried oxide, is fabricated with $193 \mathrm{~nm}$ DUV lithography through the ePIXfab silicon photonic platform. The III$\mathrm{V}$ membrane has a total thickness of $583 \mathrm{~nm}$ and includes 3 compressively strained quantum wells that provide the 
TE mode gain and a tunnel junction for a low loss p-contact. Figure 1(a) shows schematic drawings of the entire circuit and the bonded microdisk lasers. The light from the lasers is coupled to straight SOI waveguides, which are then coupled to single mode fiber using grating couplers.

The AOFF operation is based on the switching between clockwise (cw) and counter clockwise (ccw) whispering gallery modes. To obtain unidirectional operation ( $\mathrm{cw}$ or $\mathrm{ccw}$ ), the coupling between the $\mathrm{cw}$ and $\mathrm{ccw}$ modes must be very small and the internal power density must be sufficiently high [7]. In our microdisks, large internal power density results from the good mode confinement of the InP membrane due to the high index contrast. We also avoided degradation due to heating at higher currents, by making the Au layer of the top contact thick (600nm) and using it as heat sink. Since the whispering gallery mode is confined to the edge of the disk, this top metal does not result in substantial optical absorption loss. The coupling between $\mathrm{cw}$ and $\mathrm{ccw}$ modes was minimized by reducing the surface sidewall roughness using an optimized lithography and etching process. Figure 1(b) shows the light power measured from the lhs and rhs grating couplers as a function of the injected current. Unidirectional operation is obtained for currents above $1.7 \mathrm{~mA}$. Due to their small dimensions, the spectrum of the laser light shows a good single mode behavior with a side mode rejection above $40 \mathrm{~dB}$ and free spectral range of $30 \mathrm{~nm}$.

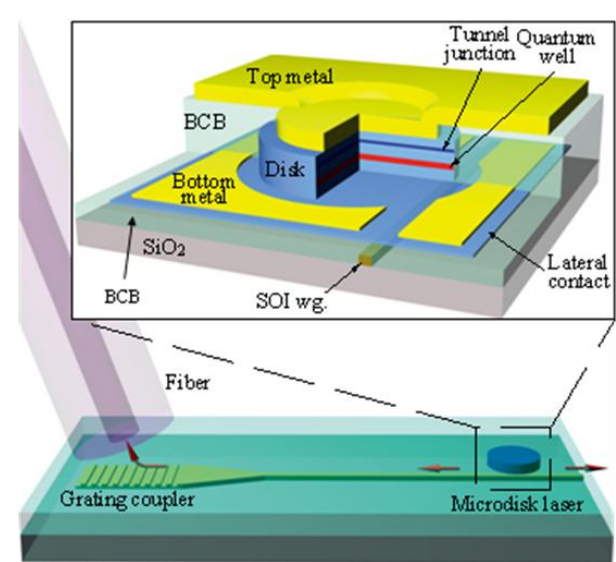

(a)

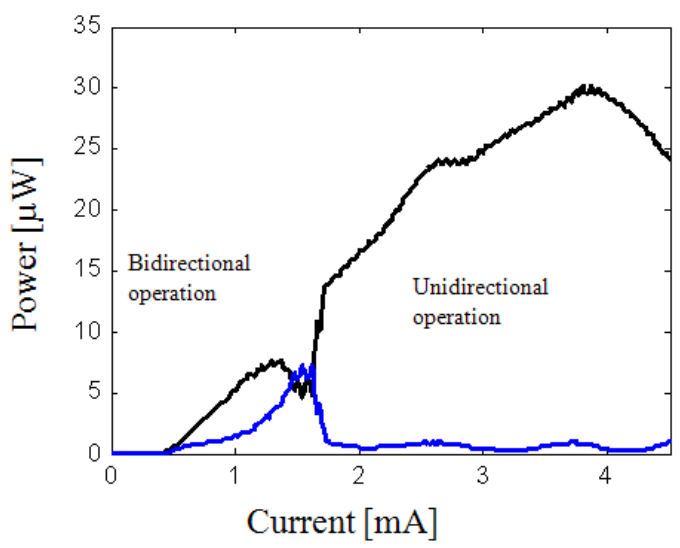

(b)

Fig. 1. (a) Schematic structure of the InP microdisk laser coupled to an SOI waveguide and detail of the microdisk laser (inset) and (b) power inthe clockwise (black) and counter clockwise (blue) whispering gallery mode measured for a $7.5 \mu \mathrm{m}$ diameter disk. (From [4])

The same microdisk lasers can be used for all-optical gating or wavelength conversion. When used as all-optical gates, the microdisks are pumped below their threshold current or can be even without or with negative bias. The operation is based on shifts of the resonance frequency of the microdisk resonators by changing the carrier density and refractive index with an optical signal. The weak optical clock signal can then be routed to either the drop or through port. All-optical wavelength conversion is again achieved by biasing the microdisk lasers above threshold. A modulation at a pump wavelength, which corresponds with a nondominant lasing mode, is transferred to an inverted modulation at the dominant laser mode.

\section{Switching Experiments}

Switching between $\mathrm{cw}$ and ccw operation in the microdisk based AOFF is achieved through short pulses that are injection locking the laser. For the switching experiments, we biased the laser at $3.5 \mathrm{~mA}$, which is about twice the threshold for the unidirectional operation $(1.7 \mathrm{~mA})$ and is chosen to avoid noise-induced switching. To test the switching speed and the switching energy, we used 100 ps long set and reset pulses as shown in Figure 2(a) and measured the laser output (shown in Figure 2(b)) with a high speed oscilloscope. Because the disk is only coupled to one straight waveguide, it is not possible to separate the switch pulses and the laser signal. Therefore the switch pulses always cover the transient of the microdisk signal, making it difficult to measure the exact switching times. 
The residual reset pulses also come from the reflection at the cleaved facet of the access fiber and have been suppressed to some extent by using index matching fluid. The measured switch-off transient in this case is shown in Figure 2(c); it is characterized by a switch-off time of 60ps. The peak power measured in the SOI waveguide was 18 $\mu \mathrm{W}$, corresponding with a switching energy of $1.8 \mathrm{fJ}$.
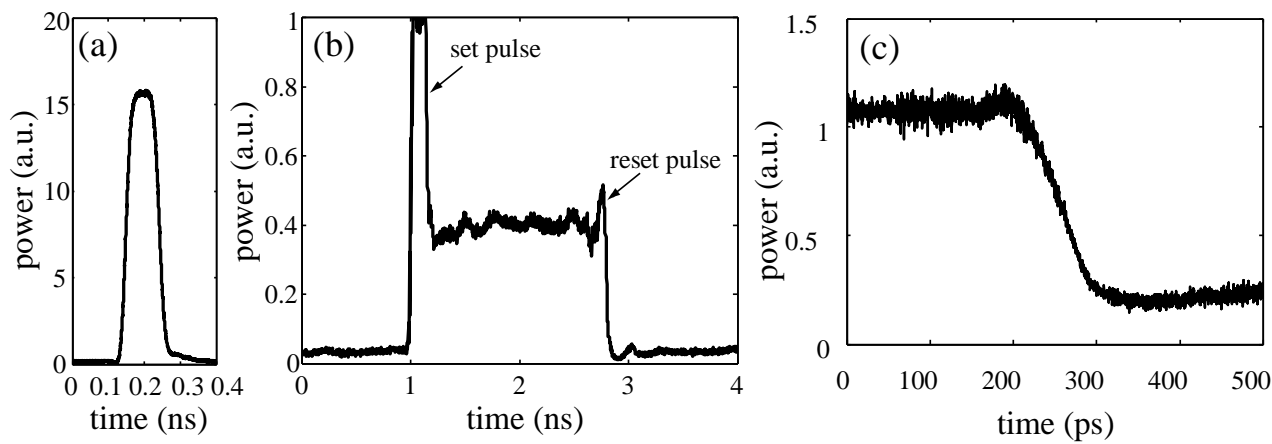

Fig. 2. (a) Waveform of the injected optical pulse, (b) Waveform of the measured optical signal at one side, (c) Details of the switch-off transient, after applying the index matching fluid to suppress the appearance of the reset pulses. (From [4]).

\section{Other optical logic gates}

Using the microdisk based gates and set-reset AOFF, other sequential logic devices such as D-flip-flops, shift registers, etc. can be readily conceived. Furthermore, combinatorial logic gates such as OR, NAND, NOR gates can be designed when combining microdisk lasers with Bragg reflectors in the SOI waveguides. The SOI circuits for several of these gates are being fabricated and results will be given at the conference.

\section{Conclusion}

Using the heterogeneous integration of InP membranes onto SOI passive waveguide circuits, we have demonstrated compact, low power and high speed optical logic devices such as set-reset flip-flops, gates and wavelength converters. These first demonstrations pave the way towards the realization of more complex photonic integrated circuits implementing more logic operations with low power and small footprint. Demonstration of other logic functions and new optimized designs are planned and will be reported on during the conference.

\section{Acknowledgement}

This work is supported by the European FP7 ICT-projects HISTORIC, WADIMOS and PhotonFab, the Fund for Scientific Research Flanders (FWO), and the IAP-project "Photonics@be". The work of K. Huybrechts and T. Spuesens is supported by the Institute for the Promotion of Innovation through Science and Technology (IWT) under a specialization grant. G. Roelkens acknowledges the Fund for Scientific research Flanders for a post-doctoral grant. We acknowledge the assistance from Steven Verstuyft during the device fabrication.

\section{References}

1. R. Tucker, "Role of Optics and Electronics in High-Capacity Routers", IEEE J. Lightwave Technol. 24, 4655-4673 (2006).

2. J. Van Campenhout, et al., "Electrically pumped InP-based microdisk lasers integrated with a nanophotonic silicon-on-insulator waveguide circuit," Optics Express., vol. 15, 6744-6749 (2007).

3. G. Roelkens, et al, "III-V/Si photonics by die-to-wafer bonding", Materials Today, 10 (7-8), 36-43 (2007).

4. L. Liu, et al, "An ultra-small, low-power, all-optical flip-flop memory on a silicon chip”, Nature Photonics, Vol. 4, Nr. 3, pp. 182-187, March 2010.

5. R. Kumar, et al, "10GHz All-Optical Gate on a Silicon Chip", paper JWA44, Proc. of OFC, San Diego, March 2010.

6. O. Raz, et al., "Compact, Low Power and Low Threshold Electrically Pumped MicroDisc Lasers for $20 \mathrm{~Gb} / \mathrm{s}$ Non Return to Zero AllOptical Wavelength Conversion”, paper OMQ5, Proc. of OFC, San Diego, March 2010.

7. G. Mezösi, et al, "Unidirectional Bistability in AlGaInAs Microring and Microdisk Semiconductor Lasers," PTL21, 88-90 ( 2009). 